\title{
Photoluminescence of Mg-doped m-plane GaN grown by MOCVD on bulk GaN substrates
}

\author{
Bo Monemar, Plamen Paskov, Galia Pozina, Carl Hemmingsson, Peder Bergman, \\ David Lindgren, Lars Samuelson, Xianfeng Ni, Hadis Morkoç, Tanya Paskova, \\ Zhaoxia Bi and Jonas Ohlsson
}

\section{Linköping University Post Print}

\begin{abstract}
N.B.: When citing this work, cite the original article.
\end{abstract}
Original Publication:

Bo Monemar, Plamen Paskov, Galia Pozina, Carl Hemmingsson, Peder Bergman, David Lindgren, Lars Samuelson, Xianfeng Ni, Hadis Morkoç, Tanya Paskova, Zhaoxia Bi and Jonas Ohlsson, Photoluminescence of Mg-doped m-plane GaN grown by MOCVD on bulk GaN substrates, 2011, Proc. SPIE 7939, 793907.

http://dx.doi.org/10.1117/12.874687

Copyright: Society of Photo-optical Instrumentation Engineers

Postprint available at: Linköping University Electronic Press

http://urn.kb.se/resolve?urn=urn:nbn:se:liu:diva-73568 


\title{
Photoluminescence of Mg-doped $m$-plane GaN grown by MOCVD on bulk GaN substrates
}

\author{
Bo Monemar*a,b, Plamen Paskov ${ }^{\mathrm{a}}$, Galia Pozina ${ }^{\mathrm{a}}$, Carl Hemmingsson ${ }^{\mathrm{a}}$, Peder Bergman ${ }^{\mathrm{a}}$, David \\ Lindgren $^{\mathrm{b}}$, Lars Samuelson ${ }^{\mathrm{b}}$, Xianfeng $\mathrm{Ni}^{\mathrm{c}}$, Hadis Morkoç ${ }^{\mathrm{c}}$, Tanya Paskova ${ }^{\mathrm{d}}$, Zhaoxia $\mathrm{Bi}^{\mathrm{e}}$, and Jonas \\ Ohlsson ${ }^{\mathrm{e}}$ \\ ${ }^{a}$ Dept of Physics, Chemistry and Biology, Linköping University, S-581 83 Linköping, Sweden \\ ${ }^{b}$ Solid State Physics-The Nanometer Structure Consortium, Lund University, Box 118, S-221 00 \\ Lund, Sweden \\ ${ }^{\mathrm{c}}$ Dept of Electrical and Computer Engineering, Virginia Commonwealth University, Richmond, VA \\ 23284-3072, USA \\ ${ }^{\mathrm{d}}$ Kyma Technologies Inc., Raleigh, North Carolina 27617, USA \\ ${ }^{e}$ Glo AB, Ideon Science Park, Scheelevägen 17, S-223 70 Lund, Sweden
}

\begin{abstract}
Photoluminescence (PL) properties are reported for a set of $m$-plane GaN films with Mg doping varied from mid $10^{18}$ $\mathrm{cm}^{-3}$ to well above $10^{19} \mathrm{~cm}^{-3}$. The samples were grown with MOCVD at reduced pressure on low defect density m-plane bulk GaN templates. The sharp line near bandgap bound exciton (BE) spectra observed below $50 \mathrm{~K}$, as well as the broader donor-acceptor pair (DAP) PL bands at $2.9 \mathrm{eV}$ to $3.3 \mathrm{eV}$ give evidence of several Mg related acceptors, similar to the case of c-plane GaN. The dependence of the low temperature BE spectra on excitation intensity as well as the transient decay behavior demonstrate acoustic phonon assisted transfer between the acceptor BE states. The lower energy donor-acceptor pair spectra suggest the presence of deep acceptors, in addition to the two main shallower ones at about $0.23 \mathrm{eV}$. Similar spectra from Mg-doped GaN nanowires (NWs) grown by MOCVD are also demonstrated and briefly discussed.
\end{abstract}

Keywords: GaN, $m$-plane, MOCVD, Mg-doping, photoluminescence, nanowires

\section{INTRODUCTION}

Non-polar or semi-polar surface orientations are currently of high interest for development of III-nitride based light emitting diodes (LEDs), since these orientations allow the elimination of the polarization-induced internal electric fields in the quantum wells (QWs) of planar InGaN LED structures ${ }^{1}$. This in turn should provide an increased radiative efficiency of such QW structures, as well as a flexibility in designing the width of the QWs ${ }^{2}$. It is less clear how doping can be controlled for such growth surfaces. It is of basic interest to explore whether the same doping configurations are incorporated in growth on different facets, and also whether the incorporation rates differ. Previous work on non-polar epi-layers shows a high structural defect density in the case of epitaxial growth on foreign substrates ${ }^{3}$. In order to study optical spectra of doped epilayers or QW structures it is then necessary to use samples grown on high quality bulk GaN substrates, to avoid strong interference with spectra caused by stacking fault (SF) defects ${ }^{4}$. This is especially true for photoluminescence (PL) spectra of acceptor-doped layers, where the PL emissions strongly overlap with SF-related spectra in the photon energy range between $3.2 \mathrm{eV}$ and the $\mathrm{GaN}$ bandgap.

In this work we have studied Mg-doping in $m$-plane GaN epi-layers grown by Metal Organic Chemical Vapor Deposition (MOCVD). The PL spectra have been studied as a function of doping concentration and measuring temperature, and compared with previous data on similar $c$-plane samples. In addition we have studied Mg-doping in regular GaN nano-wire (NW) structures, which have $m$-plane side facets. The results demonstrate that PL appears to be a useful characterization technique for $\mathrm{Mg}$-doping in such nanostructures.

*bom@ifm.liu.se, Phone: +4613 281765, Fax: +4613137568 


\section{SAMPLES AND EXPERIMENTAL PROCEDURES}

\subsection{Growth conditions}

$500 \mathrm{~nm}$ thick $\mathrm{Mg}$-doped $m$-plane GaN films were grown on bulk $m$-plane GaN substrates under 400 Torr chamber pressure at $\sim 1000-1010^{\circ} \mathrm{C}$, using MOCVD. A $1.5 \mu \mathrm{m}$ thick undoped GaN buffer layer was grown on top of the bulk substrate before the deposition of the Mg-doped layer. Trimethylgallium (TMGa) and ammonia were used as the Ga and $\mathrm{N}$ sources with a flow rate of $54 \mu \mathrm{mol} / \mathrm{min}$, and $7 \mathrm{SLM}$ (standard liter per min), respectively. The $\mathrm{Mg}$ doping concentration $([\mathrm{Mg}])$ was varied by changing the $\mathrm{Cp}_{2} \mathrm{Mg}$ source flow rate from $0.24 \mu \mathrm{mol} / \mathrm{min}$ to $7.2 \mu \mathrm{mol} / \mathrm{min}$. The samples discussed here have Mg concentrations $3 \times 10^{18} \mathrm{~cm}^{-3}(\mathrm{~A}), 8 \times 10^{18} \mathrm{~cm}^{-3}(\mathrm{~B}), 1 \times 10^{19} \mathrm{~cm}^{-3}(\mathrm{C})$, and $2.5 \times 10^{19} \mathrm{~cm}^{-3}(\mathrm{D})$, as measured by SIMS data (Evans Analytical Group). As a comparison, an undoped MOCVD-grown $500 \mathrm{~nm} m$-plane $\mathrm{GaN}$ film on bulk $m$-GaN was used as a reference sample. The $m$-plane freestanding GaN substrates, provided by Kyma Technologies, were nominally undoped and had a threading dislocation density $<5 \times 10^{6} \mathrm{~cm}^{-2}$. Their off-cut is $0.11^{\circ}$ $0.15^{\circ}$ towards the GaN $a$-axis and $0.13^{\circ}-0.21^{\circ}$ towards the $\mathrm{GaN}+c$-axis. The NW samples are grown with MOCVD at GLO AB, using a Thomas Swan close-coupled showerhead growth chamber.

\subsection{Annealing and optical measurements}

Each of the planar samples was cut in two pieces, and one piece was furnace annealed for about 10 min at $800^{\circ} \mathrm{C}$ in flowing $\mathrm{N}_{2}$ gas. Stationary PL spectra for the planar $m$-plane samples were measured with cw UV excitation (photon energy of $4.66 \mathrm{eV}$ or $3.81 \mathrm{eV}$ ), from $2 \mathrm{~K}$ to $300 \mathrm{~K}$. The NW structures were studied with a micro-PL setup, where stationary PL spectra from individual NWs as well as an NW ensemble could be measured between 2 K and $300 \mathrm{~K}$. PL transient measurements were done using femtosecond pulses from a frequency tripled Ti:sapphire laser $(76 \mathrm{MHz}, 4.66$ $\mathrm{eV}$ ), and detected with a UV sensitive Hamamatsu streak camera with a fast sweep unit.

\section{EXPERIMENTAL RESULTS}

\subsection{Photoluminescence spectra of planar $m$-plane epitaxial layers}

Fig. 1 shows a PL spectrum at $2 \mathrm{~K}$ for a nominally undoped $m$-plane reference sample, grown in the same MOCVD equipment as the $\mathrm{Mg}$-doped samples. At high energies the free excitons (denoted $\mathrm{X}_{\mathrm{A}}$ and $\mathrm{X}_{\mathrm{B}}$, respectively) are observed. The main PL peaks are due to neutral donor bound excitons $\left(\mathrm{Si}^{\circ} \mathrm{X}_{\mathrm{A}} \text { and } \mathrm{O}^{\circ} \mathrm{X}_{\mathrm{A}} \text {, respectively) }\right)^{5}$. The strongest line is related to the $\mathrm{O}$ donors, consistent with previous observations of a stronger incorporation of residual $\mathrm{O}$ in $m$-plane,

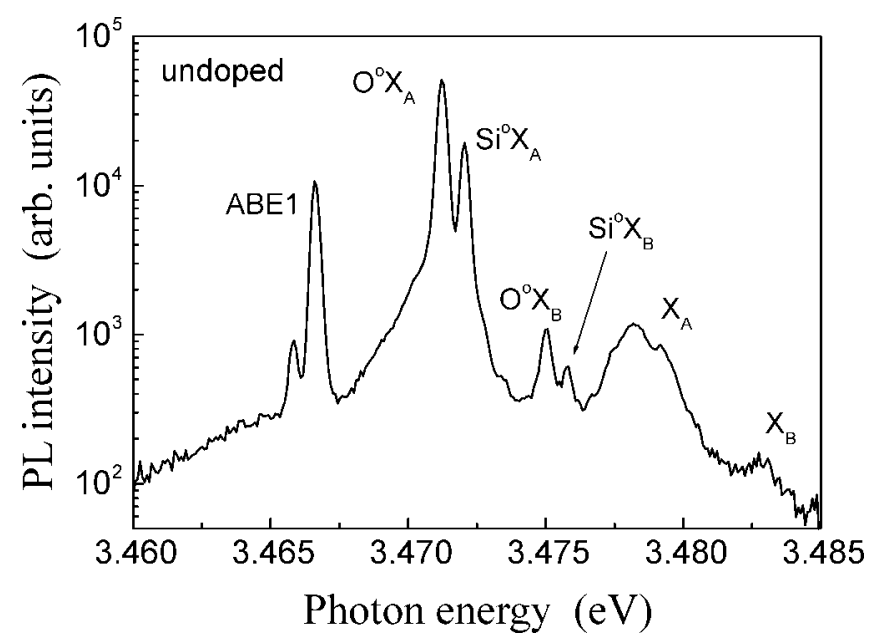

Figure 1 Low-temperature (2 K) PL spectrum in the near bandgap region of a nominally undoped $m$-plane epilayer. 
compared to $c$-plane growth ${ }^{6}$. The observed linewidth of the BE lines $(<1 \mathrm{meV})$ indicate a residual donor density of $<$ about $1 \times 10^{17} \mathrm{~cm}^{-3}$. The doublet line $\mathrm{ABE} 1$ is due to $\mathrm{Mg}$ contamination in the growth system. It was recently demonstrated that acceptor BEs (ABEs) in wurtzite wide bandgap semiconductors are expected to exhibit several radiative states $^{7}$.

Fig. 2 shows low temperature stationary PL spectra in the near bandgap region for sample A. The free exciton (FE) and donor bound exciton (DBE) peaks are weak, as expected for rather heavy Mg-doping. A dominant peak at $3.466 \mathrm{eV}$ is the Mg-related A1 acceptor BE peak (ABE1), the same as observed in Fig. $1^{8}$. This peak has a low energy wing due to acoustic phonon coupling, common for ABEs in wide bandgap materials. A broad second ABE peak (ABE2) related to acceptor $\mathrm{A} 2$ is seen at $3.454 \mathrm{eV}^{2}$, this peak increases superlinearly in intensity with increased excitation. This broad feature shows some structure, a peak at $3.458 \mathrm{eV}$ is observed in the figure. The broad ABE2 peak shows a similar appearance as for c-plane Mg-doped GaN previously studied. The reason for this broadening might be an acoustic phonon assisted transfer process of excitons from ABE1 states to ABE2. The acoustic phonons in GaN cover an energy range up to $>20 \mathrm{meV}$ in $\mathrm{GaN}^{9}$. The $\mathrm{ABE} 2$ peak shows a strong dependence of excitation intensity (Fig. 2), possibly related to the hole occupation of A2 (quasi Fermi level position) versus excitation. A weaker peak (X) at $3.444 \mathrm{eV}$ with a low energy acoustic phonon wing completes the near bandgap spectrum. This peak was not observed in our previous studies on $\mathrm{Mg}$-doping in $c$-plane $\mathrm{GaN}^{8}$. A possible explanation of this weak peak could be a deep acceptor bound exciton.

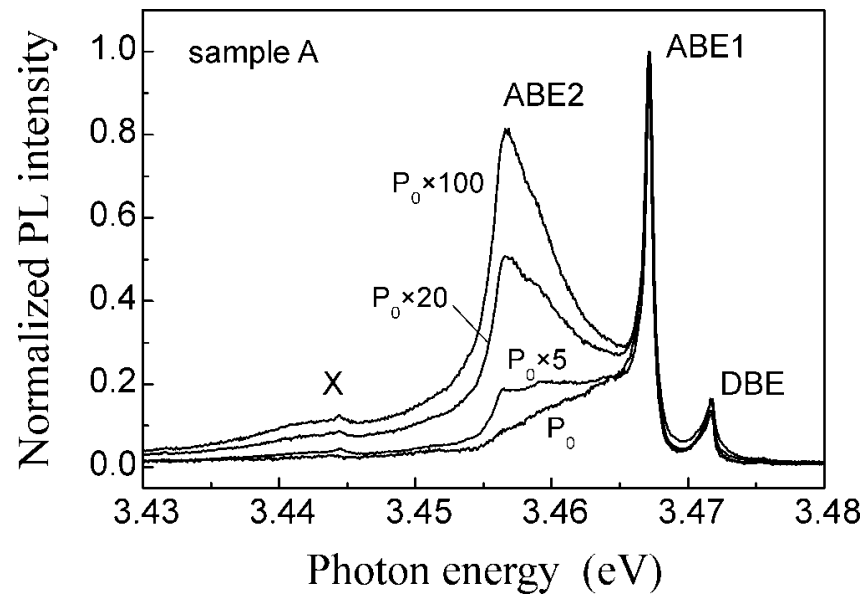

Figure 2 Near bandgap PL spectra of sample A taken at $2 \mathrm{~K}$ and different excitation power.

There is no strong difference in PL spectra between virgin samples and annealed samples for this series, presumably due to a significant activation of the acceptors during growth and cool down, i. e. the H passivation is largely removed during cooling from the growth temperature. In Fig. 3 is shown a low temperature PL spectrum for sample B in the near bandgap region, before and after anneal. The ABE2 peak is more dominant after anneal, similar to the case of $c$-plane growth $^{8}$. An interesting observation is the presence a several very strong lines in the spectral range $3.38 \mathrm{eV}-3.42 \mathrm{eV}$. These lines were earlier assigned to discrete donor-acceptor pair lines ${ }^{10}$, which however is inconsistent with the known binding energy of the $\mathrm{A} 1 \mathrm{Mg}$ acceptor ${ }^{11}$. The lines exist in c-plane samples as well, and are likely to be $\mathrm{Mg}$-related. $\mathrm{A}$ possible assignment is $\mathrm{BEs}$ related to complex $\mathrm{Mg}$-related defects.

Fig. 4 shows the PL spectra at $2 \mathrm{~K}$ at lower photon energies, in the region of the DAP recombination, for four samples. The structured PL emission with a no-phonon (NP) line at $3.27 \mathrm{eV}$ has been correlated to $\mathrm{Mg}$-acceptors ${ }^{12}$, in our recent work it has been argued that this emission is a fingerprint of the $\mathrm{A} 1 \mathrm{Mg}$ acceptor ${ }^{8}$. The dominant $\mathrm{Mg}$-related $3.27 \mathrm{eV}$ DAP emission with its LO phonon replicas is present in all samples, but broad emission bands at lower energies are dominant for the highest doping (Fig. 4). This picture is very similar to what has been found for $c$-plane Mg-doped $\mathrm{GaN}^{13}$. 


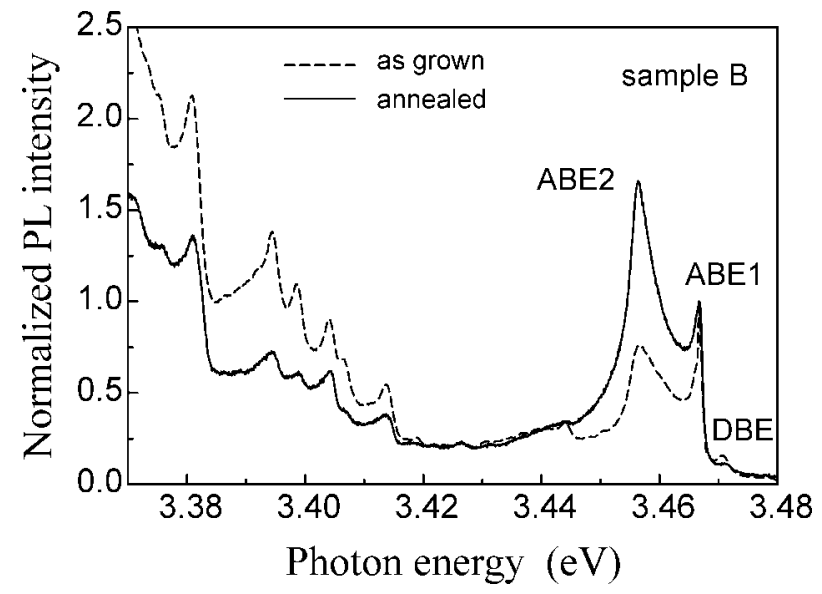

Figure 3 Low-temperature (2 K) PL spectra of sample B before and after annealing.

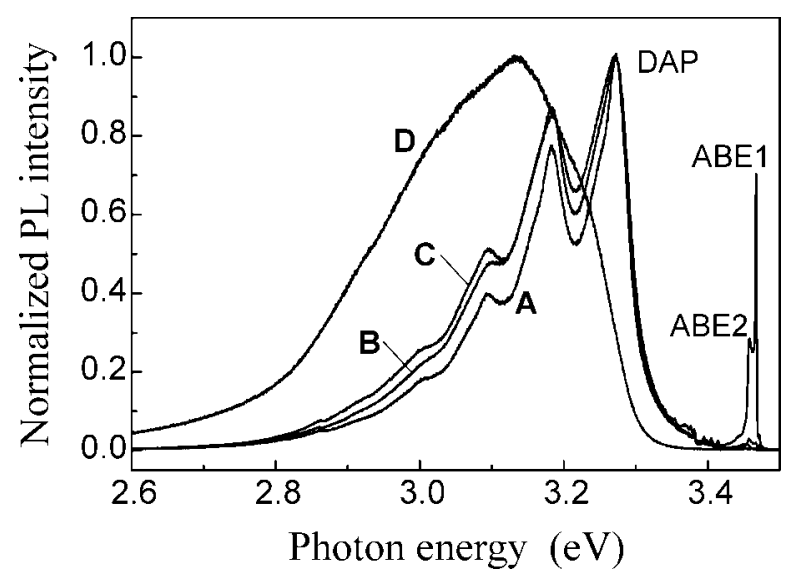

Figure 4 Low-temperature (2 K) PL spectra of four samples after annealing.

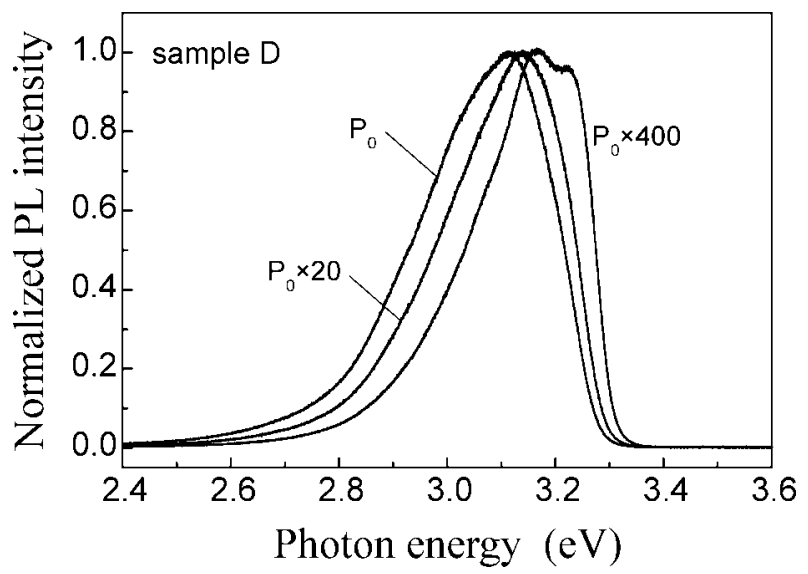

Figure 5 Low-temperature $(2 \mathrm{~K})$ PL spectra of sample D at different excitation power. 
Fig. 5 shows an example of the excitation intensity dependence of the PL spectrum in the DAP region for sample D. This

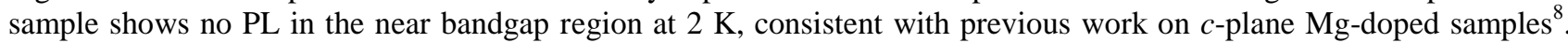
Excitonic spectra are not observed at these high doping densities. At low excitation the main PL peak occurs around 3.1 $\mathrm{eV}$ (presumably mainly related to the acceptor A2), while at the highest excitation the A1-related 3.27 eV DAP peak is enhanced. This behavior is explained mainly by saturation of DAP emissions involving deeper acceptor states, which have strong phonon coupling and a lower oscillator strength for the DAP emission ${ }^{11}$. It is assumed that the main donors present are shallow $\mathrm{O}$ and $\mathrm{Si}$ donors ${ }^{11}$. We suggest that the deeper PL emission peaking around $3.0 \mathrm{eV}$ (Fig. 4) is related to DAPs involving deep Mg-related acceptors and shallow donors (in the case of m-plane samples mainly $\mathrm{O}$ donors) ${ }^{11}$.

\subsection{Temperature dependence}

Fig. 6 shows near bandgap PL spectra of the lowest doped sample A at some different cryogenic temperatures. The ABE1 and ABE2 features are largely quenched at $40 \mathrm{~K}$, while the weaker $3.444 \mathrm{eV}$ peak survives up to about $50 \mathrm{~K}$. $\mathrm{ABEs}$ in $\mathrm{GaN}$ are known to be quenching at these low temperatures ${ }^{5}$, the somewhat higher stability of the $3.444 \mathrm{eV}$ peak (X) is consistent with its stronger BE binding energy. The origin of this peak is not identified, it could be related to a deeper Mg-related acceptor than A1 and A2, but it may also be related to a structural defect. This PL feature was not observed in $c$-plane Mg-doped samples ${ }^{8,13,14}$.

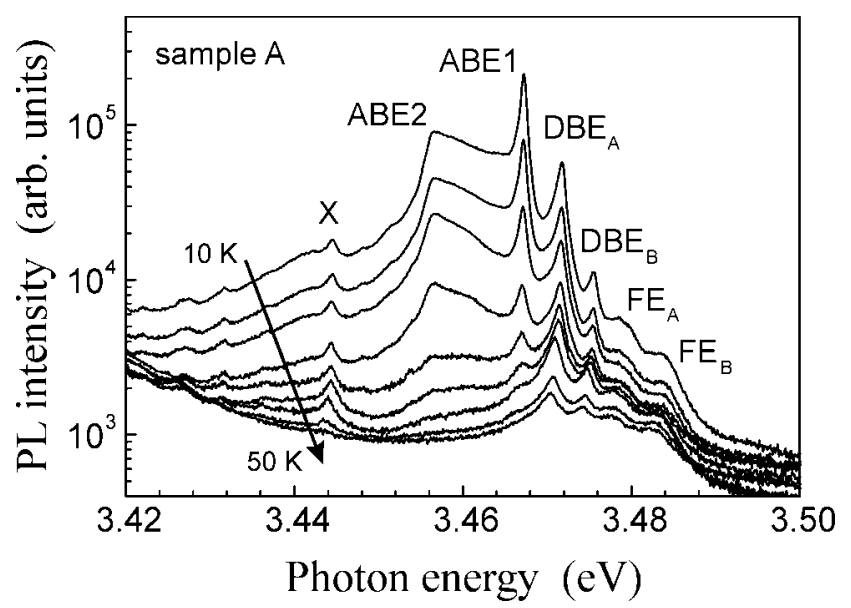

Figure 6 Temperature dependent PL spectra of sample A in the near bandgap region.

\subsection{Transient PL data and decay times}

In previous work it was determined that the radiative decay time of ABE1 is about $900 \mathrm{ps}^{5}$, as obtained from low doped $\mathrm{n}$-type $c$-plane samples. For higher doping conditions, as for the $\mathrm{Mg}$-doping in these $m$-plane samples exciton transfer processes affect the decay times substantially ${ }^{5,14}$. Fig. 7 shows decays for the ABE region for sample B. The ABE1 shows a non-exponential decay with a fast initial part about $400 \mathrm{ps}$, and a slower later part. This faster decay is interpreted as evidence of exciton transfer from ABE1 to the lower broad ABE2 spectrum and also to the X-line. The X peak at $3.444 \mathrm{eV}$ clearly has a different longer decay time.

The DAP recombination appearing at lower photon energies in the PL spectra have a much longer decay time, as noted previously for $c$-plane $\mathrm{Mg}$-doped $\mathrm{GaN}^{8}$. In Fig. 8 we show time resolved PL (TRPL) spectra for sample D at $2 \mathrm{~K}$. Initially the $2.27 \mathrm{eV}$ DAP spectrum related to the $\mathrm{A} 1 \mathrm{Mg}$ acceptor dominates, but after several $\mu$ s delay a broad peak centered at about $3.15 \mathrm{eV}$ dominates, presumably related to the $\mathrm{A} 2 \mathrm{Mg}$ acceptor ${ }^{8}$. There is also a broad signal towards lower photon energies, indicating the presence of deeper acceptors ${ }^{11}$. These TRPL data are very similar to the ones previously obtained for $c$-plane $\mathrm{Mg}$-doped $\mathrm{GaN}^{8}$. 

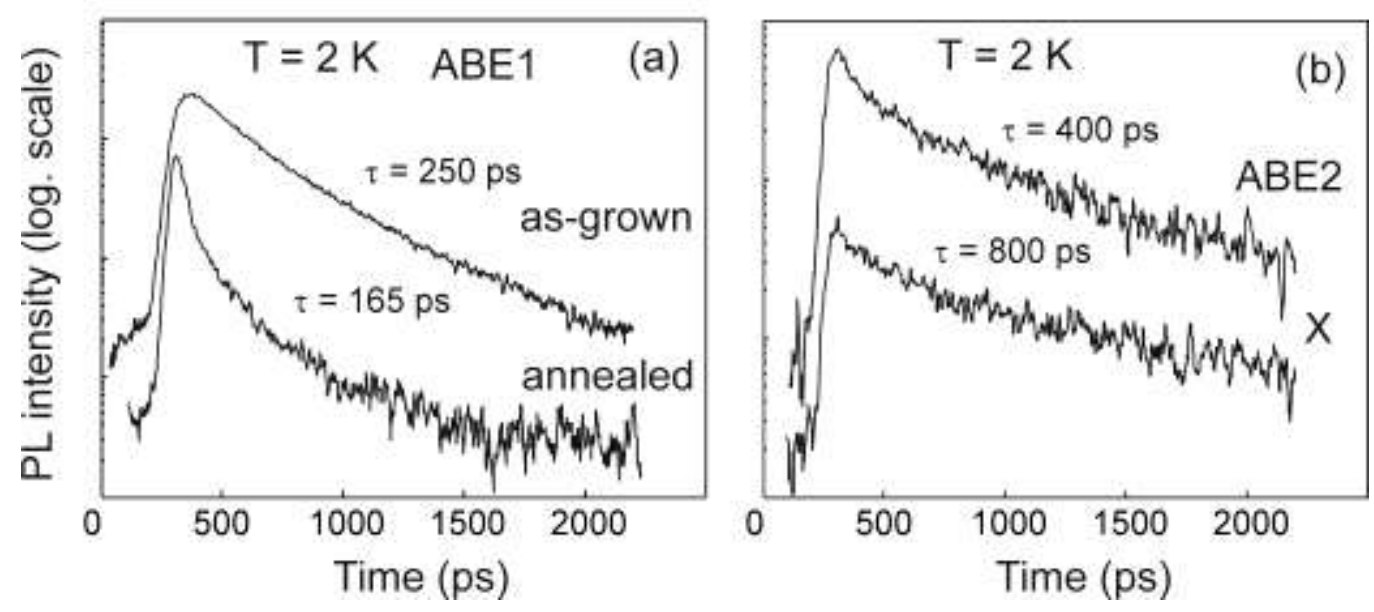

Figure 7 PL decay curves taken at $2 \mathrm{~K}$ for GaN doped with Mg concentration of $1 \times 10^{19} \mathrm{~cm}^{-3}$ (sample C).

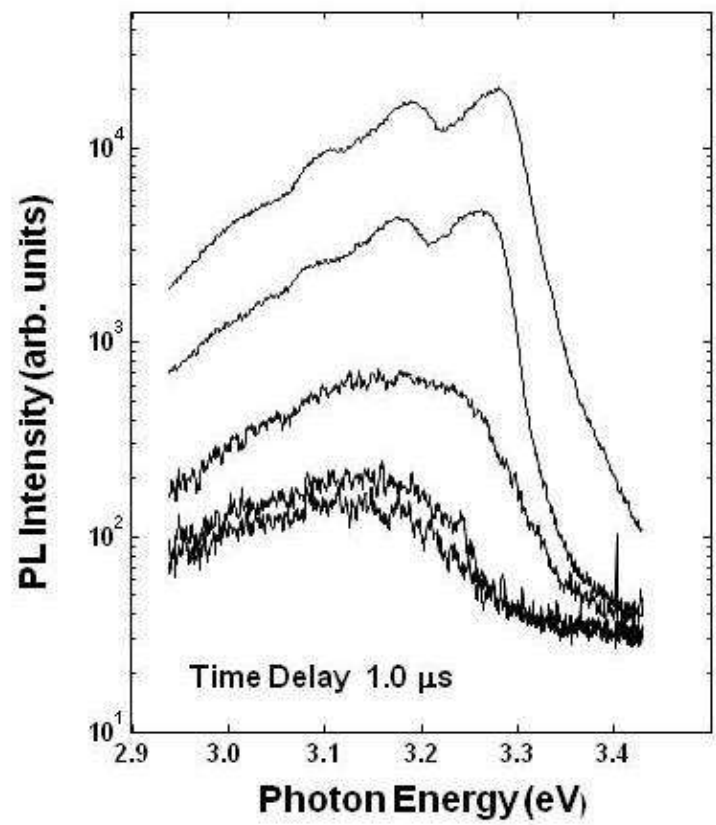

Figure. 8 Time-resolved PL spectra for sample D, obtained with femtosecond excitation pulses with $250 \mathrm{kHz}$ repetition rate and a time delay of $1 \mu$ s between each curve.

\subsection{Mg-doped GaN nanowires}

Fig 8 shows NW array PL spectra as well as micro-PL spectra for two single NWs of about $400 \mathrm{~nm}$ diameter with $\mathrm{Mg}$ doping density estimated as high $10^{18} \mathrm{~cm}^{-3}$ and high $10^{19} \mathrm{~cm}^{-3}$, respectively. The NWs were Mg-doped during growth, and the PL mainly originates from the m-plane side facets of the NWs (see Fig 9). In the low doped sample the $3.27 \mathrm{eV}$ DAP emission dominates, while PL in the highly doped sample is broader and peaks at lower energy. This is similar to the data for the epi-layers in Fig. 4, and demonstrates the feasibility of controlling Mg-doping in the nanowire growth process. 

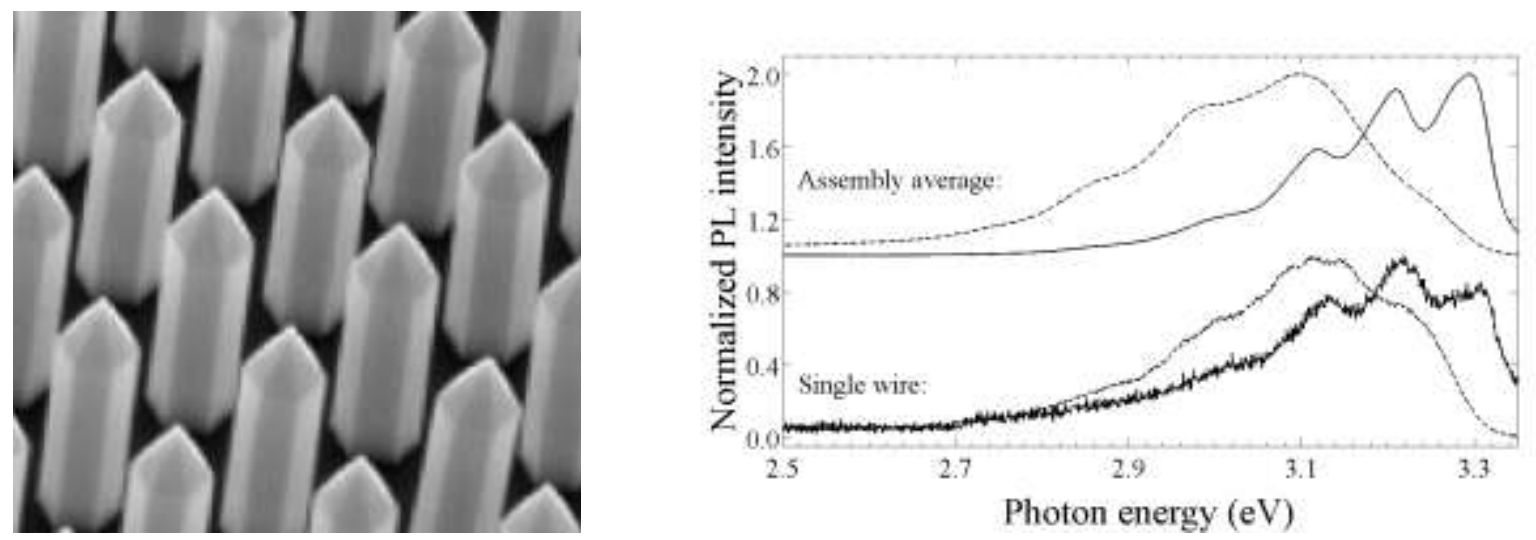

Figure 9. SEM picture of a GaN nano-wire assembly (left). Micro-PL spectra in the DAP region of similar Mg-doped GaN NW samples (right), taken both for an assembly of NWs (upper part) and single wires (lower part). Spectra for low ( - ) as well as high ( - - ) Mg doping (see text) are shown.

\section{DISCUSSION}

The PL spectra for $m$-plane Mg-doped samples are essentially very similar to the corresponding ones for $c$-plane growth 8,11 . This is expected unless specific defect reactions occur on the $m$-plane surface during the growth procedure. The incorporation of $\mathrm{Mg}$ acceptors in m-plane MOCVD growth has been reported to be somewhat lower than in c-plane growth under comparable growth conditions ${ }^{6}$. This appears consistent with our SIMS data. Under the growth conditions for these samples acceptor activation seems to be largely completed after cool down from growth. The main new spectral feature in the Mg-doped $m$-plane samples seems to be a weak peak X at $3.444 \mathrm{eV}$, with an associated acoustic phonon wing. This peak is so far unidentified. The broad spectral features for the $3.454 \mathrm{eV}$ ABE2 PL peak may be understood considering an acoustic phonon assisted transfer of excitons to the ABE2 state, occurring on the same time scale as the $\mathrm{ABE}$ recombination. The appearance of the DAP spectra in the range $2.9 \mathrm{eV}$ to $3.3 \mathrm{eV}$ is similar to the corresponding results for the $c$-plane $\mathrm{Mg}$-doped samples studied earlier ${ }^{8,11}$. As in $c$-plane growth there seem to be several Mg-related acceptors present, some are considerably deeper than $0.2 \mathrm{eV}$, and these are expected to influence the position of the Fermi level in the case of low residual donor concentrations ${ }^{11}$. The PL spectra for Mg-doped NW samples are consistent with the set of planar epi-layers studied, as expected since the $m$-plane facets are dominating the emission from these NWs.

\section{ACKNOWLEDGMENTS}

We are grateful to the K. A. Wallenberg Foundation for the financing of equipment for the laser spectroscopy, and to the Swedish Energy Agency for a Project Grant.

\section{REFERENCES}

[1]. Paskova, T. (editor), Nitrides with Non-polar Surfaces: Growth, Properties and Devices (Wiley-VCH, Weinheim, 2008).

[2]. Speck, J. S. and Chichibu, S. F., MRS Bulletin 34, 304 (2009)

[3]. Paskov, P. P., Schifano, R., Monemar, B., Paskova, T., Figge, S., and Hommel, D., J. Appl. Phys. 98, 093519 (2005).

[4]. Paskov, P. P. and Monemar, B., in Ref. 1, Chapter 8, pp. 185- 217.

[5]. Monemar, B., Paskov, P. P., Bergman, J. P., Pozina, G., Toropov, A., Shubina, T., Malinauskas, T., and Usui, A.,Phys. Status Solidi B 2451723 (2008).

[6]. Cruz, S. C., Keller, S., Mates, T. E., Mishra, U., K., and DenBaars, S. P., J. Crystal Growth 311, 3817 (2009). 
[7]. Gil, B., Bigenwald, P., Paskov, P. P., and Monemar, B., Phys. Rev. B 81, 085211 (2010).

[8]. Monemar, B., Paskov, P. P., Pozina, G., Hemmingsson, C., Bergman, J. P. Kawashima, T., Amano, H., Akasaki, I., Paskova, T., Figge, S., Hommel, D., Usui, A., Phys. Rev. Lett. 102, 235501 (2009).

[9] Ruf, T., Serrano, J., Cardona, M., Pavone, P., Pabst, M., Krisch, M., D’Astuto, M., Suski, T., Grzegory, I., and Leszczynski. M., Phys. Rev. Lett. 86, 906 (2001).

[10]. Stepniewski, R., Wysmolek, A., Potemski, M., Lusakowski, J., Korona, K., Pakula, K., Baranowski, J. M., Martinez, G., Wyder, P., Grzegory, I., and Porowski, S., Phys. Status Solidi B 210, 373 (1998).

[11]. Monemar, B., Paskov, P. P., Pozina, G., Hemmingsson, C., Bergman, J.P., Amano, H., Akasaki, I., Figge, S., Hommel, D., Paskova, T., and Usui, A., Phys. Status Solidi C 7, 1850 (2010).

[12]. Chen, L., and Skromme, B. J., Mat. Res. Soc. Symp. Proc. 743, L11.35 (2003).

[13]. Pozina, G., Hemmingsson, C., Paskov, P. P., Bergman, J. P., Monemar, B., Kawashima, T., Amano, H., Akasaki, I., Usui, A., Appl. Phys. Lett. 82, 151904 (2008).

[14]. Monemar, B., Paskov, P. P., Bergman, J. P., Pozina, G., Toropov, A. A., Shubina, T. V., Malinauskas, T., and Usui, A., Phys. Rev. B 82, 235202 (2010). 